\title{
Surgical outcomes after cardiac surgery in liver transplant recipients
}

\author{
Takeyoshi Ota, MD, PhD, Rodolfo Rocha, MD, Lawrence M. Wei, MD, Yoshiya Toyoda, MD, PhD, \\ Thomas G. Gleason, MD, and Christian Bermudez, MD
}

\begin{abstract}
Objective: This was a single-center retrospective study to assess the surgical outcomes and predictors of mortality of liver transplant recipients undergoing cardiac surgery.
\end{abstract}

\begin{abstract}
Methods: From 2000 to 2010, 61 patients with a functioning liver allograft underwent cardiac surgery. The mean interval between liver transplantation and cardiac surgery was $5.4 \pm 4.4$ years. Of the 61 patients, $33(54 \%)$ were in Child-Pugh class A and 28 in class B. The preoperative and postoperative data were reviewed.

Results: The overall in-hospital mortality was $6.6 \%$. The survival rate was $82.4 \% \pm 5.1 \%$ at 1 year and $50.2 \% \pm 8.2 \%$ at 5 years. Cox regression analysis identified preoperative encephalopathy (odds ratio, 5.2; $95 \%$ confidence interval, $1.8-15.5 ; P=.003$ ) and pulmonary hypertension (odds ratio, $3.5 ; 95 \%$ confidence interval, 1.3-9.4; $P=.045$ ) as independent predictors of late mortality. The preoperative Model for End-Stage Liver Disease (MELD) scores of patients who died in-hospital or late postoperatively were significantly greater statistically than the scores of the others (in-hospital death, $23.7 \pm 7.8$ vs $13.1 \pm 4.5, P<.001$; late death, $15.2 \pm 6.1$ vs $12.3 \pm 4.1, P=.038)$. The Youden index identified an optimal MELD score cutoff value of 13.5 (sensitivity, 56.0\%; specificity, 67.6\%). Kaplan-Meier survival analysis successfully demonstrated that the survival rate of the MELD score less than 13.5 (MELD <13.5) group was significantly greater than that of the MELD $>13.5$ group (MELD $<13.5$ group, $93.8 \% \pm 4.2 \%$ at 1 year and $52.4 \% \pm 11.8 \%$ at 5 years; MELD $>13.5$ group, $66.9 \% \pm 9.6 \%$ at 1 year and $46.1 \% \pm 11.1 \%$ at 5 years; $P=.027$ ). In contrast, the survival rate when stratified by Child-Pugh class (class A vs B) was not significantly different.
\end{abstract}

Conclusions: Cardiac surgery in the liver allograft recipients was associated with acceptable surgical outcomes. Preoperative encephalopathy and pulmonary hypertension were independent predictors of late mortality. The cutoff value of 13.5 in the MELD score might be useful for predicting surgical mortality in cardiac surgery. (J Thorac Cardiovasc Surg 2013;145:1072-6)

The survival of liver transplant recipients has been improved by the remarkable advances in surgical technique, pre- and postoperative management, and immunosuppressive protocols, ${ }^{1}$ resulting in a potential increase in the number of patients with a history of liver transplantation requiring cardiac surgery. It has been reported that more than $70 \%$ of liver allograft recipients experience cardiac events, ${ }^{2}$ that cardiac complications after liver transplantation are common, and that these have been the leading cause of death after liver transplantation. ${ }^{3}$ Organ allograft recipients require immunosuppressant agents, placing the patients at great risk of peri- and postoperative infection. ${ }^{4}$ It has also been assumed that the liver functional reserve in liver transplantation recipients is subnormal, ${ }^{5}$ leading to

From the Division of Cardiac Surgery, Department of Cardiothoracic Surgery, University of Pittsburgh Medical Center, Pittsburgh, $\mathrm{Pa}$.

Disclosures: Authors have nothing to disclose with regard to commercial support.

Received for publication July 10, 2012; revisions received Sept 4, 2012; accepted for publication Sept 21, 2012; available ahead of print Dec 14, 2012.

Address for reprints: Christian Bermudez, MD, Division of Cardiac Surgery, Department of Cardiothoracic Surgery, University of Pittsburgh Medical Center, 200 Lothrop St, PUH C-900, Pittsburgh, PA 15213 (E-mail: bermudezc@upmc.edu). 0022-5223/\$36.00

Copyright (C) 2013 by The American Association for Thoracic Surgery http://dx.doi.org/10.1016/j.jtcvs.2012.09.099 an increased risk of surgical morbidity and mortality. Although this topic is an important entity in clinical practice, only a few of studies are available.

The purpose of the present study was to summarize our experience with surgical management of patients with a history of liver transplantation who were undergoing cardiac surgery and to determine the short- and long-term surgical outcomes and predictors of mortality.

\section{PATIENTS AND METHODS}

The quality improvement review committee of the University of Pittsburgh Medical Center approved the present study and waived the need for patient consent. From January 2000 to June 2010, 61 patients with a functioning liver allograft who were undergoing cardiac surgery at the University of Pittsburgh Medical Center were identified, and their data were retrospectively collected from our cardiac surgical database and a review of their medical records. The mean follow-up period was $3.1 \pm 2.6$ years. The follow-up was completed by telephone interview, and late outcomes were cross-referenced using the Social Security Death Index. The patient demographics are detailed in Table 1 . Their mean age was $61.5 \pm 8.2$ years. Of the 61 patients, $45(73.8 \%)$ were men; 19 patients $(31.1 \%)$ had chronic renal insufficiency preoperatively, defined as a baseline serum creatinine $>2.0 \mathrm{mg} / \mathrm{dL}$, and 13 patients $(21.3 \%)$ required dialysis. The Child-Pugh class was class A for 33 patients (54\%) and class B for 28 patients $(46 \%)$ ). No patient in Child-Pugh class $C$ underwent cardiac surgery. All patients were treated and followed up by our liver transplantation team for pre- and postoperative management. Pre- and postoperative 


\section{Abbreviations and Acronyms}

AUC = area under the curve

MELD $=$ Model for End-Stage Liver Disease

ROC $=$ receiver operating characteristic encephalopathy was diagnosed by the liver transplant team using the hepatic encephalopathy grading system. ${ }^{6}$ The mean preoperative Model for End-Stage Liver Disease (MELD) score was $13.5 \pm 5.2$. The mean interval between liver transplantation and cardiac surgery was $5.4 \pm 4.4$ years. The primary surgical indications included coronary artery disease in 31 , valvular disease in 25 (aortic valve in 17, mitral valve in 4, aortic and mitral valves in 3 , and tricuspid valve in 1 ), and other indications in 5 . Of the 61 patients, $11(18 \%)$ required emergent surgery. The indications for emergent surgery were unstable acute coronary syndrome in 6 , acute endocarditis with acute heart failure in 3, and critical aortic stenosis with progressively deteriorating hemodynamics in 2 . Six patients $(9.8 \%)$ has acute endocarditis diagnosed according to the Duke criteria. ${ }^{7}$

\section{Statistical Analysis}

Continuous variables are reported as the mean \pm standard deviation and categorical variables as frequencies. Fisher's exact test or the chi-square test was used to evaluate categorical variables on univariate analysis. Student's $t$ test or the Mann-Whitney $U$ test was used for the continuous variables owing to the distribution of the data. The Kaplan-Meier model was used to calculate the estimates for long-term survival. The logistic regression model and Cox regression model was used to determine the independent predictors of in-hospital mortality and late mortality, respectively. Only variables with $P<.05$ on univariate analysis were retained for the multivariate regression tests. For all analyses, 2-sided tests were used. The sensitivity and specificity of the preoperative MELD score as an indicator of mortality were determined from the cutoff values. Receiver operating characteristic (ROC) curves and the area under the curve (AUC) for the ROC curve were obtained by plotting the sensitivity against the falsepositive rate ( 1 - specificity). The Youden index was used to determine the optimal cutoff values of the MELD score for identification of the surgical risk of mortality (Youden index $=$ sensitivity + specificity -1 ). All statistical analyses were performed using SPSS (SPSS, Chicago, Ill).

\section{RESULTS}

The surgical procedures are summarized in Table 2. Isolated coronary artery bypass grafting was performed in 31 patients. The off-pump technique was used in $13(42 \%)$ of the isolated coronary artery bypass grafting 31 cases. Single valve replacement or repair was required in 13 cases $(21.3 \%$; aortic valve in 9 , mitral valve in 3 , and tricuspid valve in 1). Double valve surgery (aortic and mitral valves) was performed in 3 . Concomitant coronary artery bypass grafting was performed in 8 patients, along with single valve surgery (aortic valve in 6 , mitral valve in 1 , and aortic root replacement in 1).

The operative data and postoperative complications are listed in Table 3 . The mean cardiopulmonary bypass time was $130 \pm 64$ minutes. Four patients $(6.6 \%)$ required reoperation for bleeding. Eleven patients $(18.0 \%)$ experienced prolonged intubation, defined as intubation for longer than 72 hours, and 5 of these patients subsequently required tracheostomy. Seven patients ( $11.5 \%$ ) developed acute renal failure, defined as newly occurring postoperative peak serum creatinine $>2.0 \mathrm{mg} / \mathrm{dL}$. Four patients $(6.6 \%)$ newly required dialysis in the postoperative period. Postoperative infection (ie, bacteremia, pneumonia) occurred in 15 patients (24.6\%). Postoperative liver complications during the follow-up period developed in 19 patients: 7 developed ascites (5 in the postoperative in-hospital period and 2 after discharge) and 12 developed encephalopathy ( 8 in the postoperative in-hospital period and 4 after discharge). Additional liver complications occurring after discharge included drug-induced liver dysfunction in 1, bile stricture in 2 , graft rejection in 2, and recurrent hepatitis $\mathrm{C}$ in 1 patient. The graft rejection was likely because of noncompliance with the immunosuppressant therapy in 1 patient. The second patient required subsequent liver repeat transplantation about 5 months after cardiac surgery for unknown reasons.

The overall in-hospital mortality was $6.6 \%$ (4/61). The in-hospital mortality stratified by Child-Pugh class was $6.1 \%(2 / 33)$ and $7.1 \%(2 / 28)$ for class A and B, respectively $(P=1.00)$, Univariate analysis identified preoperative dialysis $(P=.008)$, serum creatinine $>2.0 \mathrm{mg} / \mathrm{dL}$ $(P=.027)$, and active endocarditis $(P=.023)$ as preoperative predictors and reoperation for bleeding $(P=.01)$, prolonged intubation $(P=.005)$, new requirement for dialysis $(P=.016)$, and new-onset encephalopathy $(P=.043)$ as postoperative predictors of in-hospital mortality. No predictor of in-hospital mortality, however, was identified on multivariate analysis. Emergent surgery was not a predictor of in-hospital or late mortality $(P=.14$ and $P=.50$, respectively). The preoperative MELD score was significantly greater for the patients who died in-hospital than for those who survived to discharge $(23.7 \pm 7.8$ vs $13.1 \pm 4.5$; $P<.001)$. The survival rate was $82.4 \% \pm 5.1 \%$ at 1 year, $71.8 \% \pm 6.3 \%$ at 3 years, and $50.2 \% \pm 8.2 \%$ at 5 years after cardiac surgery. Comparing Child-Pugh class A and $\mathrm{B}$, the survival rates were $83.5 \% \pm 6.8 \%$ at 1 year and $57.2 \% \pm 10.3 \%$ at 5 years for Child-Pugh class $\mathrm{A}$ and $81.1 \% \pm 7.6 \%$ at 1 year and $39.7 \% \pm 13.2 \%$ at 5 years for Child-Pugh class B, respectively, with no significant difference (log-rank test, $P=.603$; Figure $1, A)$. On univariate analysis, the statistically significant factors associated with late mortality were preoperative encephalopathy $(P=.012)$, serum creatinine $>2.0 \mathrm{mg} / \mathrm{dL}(P=.012)$, and pulmonary hypertension, defined as pulmonary systolic pressure $>60 \mathrm{~mm} \mathrm{Hg}(P=.016)$. The Cox regression analysis revealed preoperative encephalopathy (odds ratio, 5.2; $95 \%$ confidence interval $1.8-15.5 ; P=.003$ ) and preoperative pulmonary hypertension (odds ratio, $3.5 ; 95 \%$ confidence interval, $1.3-9.4 ; P=.045)$ as independent predictors of late mortality. The preoperative MELD score for the late mortality group was significantly greater statistically than in the survivors $(15.2 \pm 6.1$ vs $12.3 \pm 4.1$; $P=.038)$. The Youden index revealed that the optimal 
TABLE 1. Patient preoperative demographics $(n=61)$

\begin{tabular}{|c|c|}
\hline Characteristic & Value \\
\hline Age (y) & $61.5 \pm 8.2$ \\
\hline \multicolumn{2}{|l|}{ Gender } \\
\hline Male & 45 \\
\hline Female & 16 \\
\hline Diabetes mellitus & $46(75.4 \%)$ \\
\hline Smoker & $12(19.7 \%)$ \\
\hline Hypertension & $42(68.9 \%)$ \\
\hline Hyperlipidemia & $18(29.5 \%)$ \\
\hline Cerebrovascular accident & $8(13.1 \%)$ \\
\hline Chronic renal insufficiency (serum creatinine $>2.0 \mathrm{mg} / \mathrm{dL}$ ) & $19(31.1 \%)$ \\
\hline Dialysis & $13(21.3 \%)$ \\
\hline Peripheral vascular disease & $27(44.3 \%)$ \\
\hline COPD & $10(16.4 \%)$ \\
\hline \multicolumn{2}{|l|}{ Child-Pugh classification } \\
\hline A & $33(54.1 \%)$ \\
\hline B & $28(45.9 \%)$ \\
\hline $\mathrm{C}$ & $0(0 \%)$ \\
\hline MELD score & $13.5 \pm 5.2$ \\
\hline Ascites & $10(16.4 \%)$ \\
\hline Encephalopathy & $5(8.2 \%)$ \\
\hline $\mathrm{LVEF}<35 \%$ & $7(11.5 \%)$ \\
\hline Pulmonary hypertension & $8(13.1 \%)$ \\
\hline Recent myocardial infarction $(<90 \mathrm{~d})$ & $14(23.0 \%)$ \\
\hline \multicolumn{2}{|l|}{ Surgical indication } \\
\hline Coronary artery disease & $31(50.8 \%)$ \\
\hline Valvular disease & $25(41.0 \%)$ \\
\hline Other & $5(8.2 \%)$ \\
\hline Acute endocarditis & $6(9.8 \%)$ \\
\hline Emergent operation & $11(18.0 \%)$ \\
\hline Previous cardiac surgery & $5(8.2 \%)$ \\
\hline Interval between liver transplantation and cardiac surgery $(\mathrm{y})$ & $5.4 \pm 4.4$ \\
\hline
\end{tabular}

cutoff for the preoperative MELD score was 13.5 for predicting overall postoperative mortality. The ROC curve indicated that the sensitivity and specificity was 0.56 and

TABLE 2. Surgical procedures $(n=61)$

\begin{tabular}{lc}
\hline \multicolumn{1}{c}{ Procedure } & Value \\
\hline Isolated CABG & $31(50.8 \%)$ \\
Off-pump & $13 / 31$ \\
On-pump & $18 / 31$ \\
AVR & $9(14.8 \%)$ \\
MV replacement or repair & $3(4.9 \%)$ \\
TV repair & $1(1.6 \%)$ \\
AVR plus MV replacement/repair & $3(4.9 \%)$ \\
CABG plus AVR & $6(9.8 \%)$ \\
CABG plus MV replacement & $1(1.6 \%)$ \\
CABG plus aortic root replacement & $1(1.6 \%)$ \\
Aortic root replacement & $1(1.6 \%)$ \\
Other & $5(8.2 \%)$ \\
\hline Data presented as n (\%). $C A B G$, Coronary artery bypass grafting; $A V R$, aortic valve \\
replacement; $M V$, mitral valve; $T V$, tricuspid valve.
\end{tabular}

TABLE 3. Operative data, postoperative complications, and early and late mortality $(n=61)$

\begin{tabular}{lc}
\hline \multicolumn{1}{c}{ Variable } & Value \\
\hline CPB time (min) & $130 \pm 64$ \\
Required IABP & $4(6.6 \%)$ \\
Take-back for bleeding & $4(6.6 \%)$ \\
Prolonged intubation $(>72 \mathrm{~h})$ & $11(18.0 \%)$ \\
Reintubation & $9(14.8 \%)$ \\
Tracheostomy & $5(8.2 \%)$ \\
Pneumonia & $9(14.8 \%)$ \\
Bacteremia & $6(9.8 \%)$ \\
Atrial fibrillation & $21(34.4 \%)$ \\
Acute renal failure (peak creatinine $>2.0$ mg/dL) & $7(11.5 \%)$ \\
Newly required dialysis & $4(6.6 \%)$ \\
Myocardial infarction & $1(1.6 \%)$ \\
Cerebrovascular accident & $4(6.6 \%)$ \\
New-onset ascites & $7(11.8 \%)$ \\
In-hospital & $5(8.2 \%)$ \\
After discharge & $2(3.3 \%)$ \\
New-onset encephalopathy & $12(19.6 \%)$ \\
In-hospital & $8(13.1 \%)$ \\
After discharge & $4(6.6 \%)$ \\
Liver complications & $6(9.8 \%)$ \\
Peak total bilirubin $>3.0$ mg/dL & $6(9.8 \%)$ \\
In-hospital mortality & $4(6.6 \%)$ \\
Child-Pugh class A & $2 / 33(6.1 \%)$ \\
Child-Pugh class B & $2 / 28(7.1 \%)$ \\
All deaths & $26(42.6 \%)$ \\
Child-Pugh class A & $14 / 33(42.4 \%)$ \\
Child-Pugh class B & $12 / 28(42.9 \%)$ \\
Primary cause of death $(\mathrm{n}=26)$ & \\
Cerebrovascular accident & $4(6.6 \%)$ \\
Cardiac & $3(4.9 \%)$ \\
Respiratory & $5(8.2 \%)$ \\
Cancer & $2(3.3 \%)$ \\
Infection & $1(1.6 \%)$ \\
\hline Unknown Cardiopulmonary bypass; IABP, intra-aortic balloon pump. & $11(18.0 \%)$ \\
\hline
\end{tabular}

0.68 , respectively, for the optimal cutoff of 13.5. The AUC of the ROC curve was 0.633 (95\% confidence interval, 0.484-0.782). In the present cohort, 35 patients had a preoperative MELD score less than 13.5 and 26 patients a score greater than 13.5. The Kaplan-Meier survival analysis revealed that the survival rate for the MELD score $<13.5$ group was significantly greater than that for the MELD score $>13.5$ group $(93.8 \% \pm 4.2 \%$ at 1 year and $52.4 \% \pm 11.8 \%$ at 5 years for the MELD score $<13.5$ group and $66.9 \% \pm 9.6 \%$ at 1 year and $46.1 \% \pm 11.1 \%$ at 5 years for the MELD score $>13.5$ group; log-rank test, $P=.027$; Figure $1, B)$.

\section{DISCUSSION}

Liver transplantation is an established strategy to treat end-stage liver disease. It has been reported that the survival rate of liver transplant recipients is $84 \%$ at 1 year and $67 \%$ 

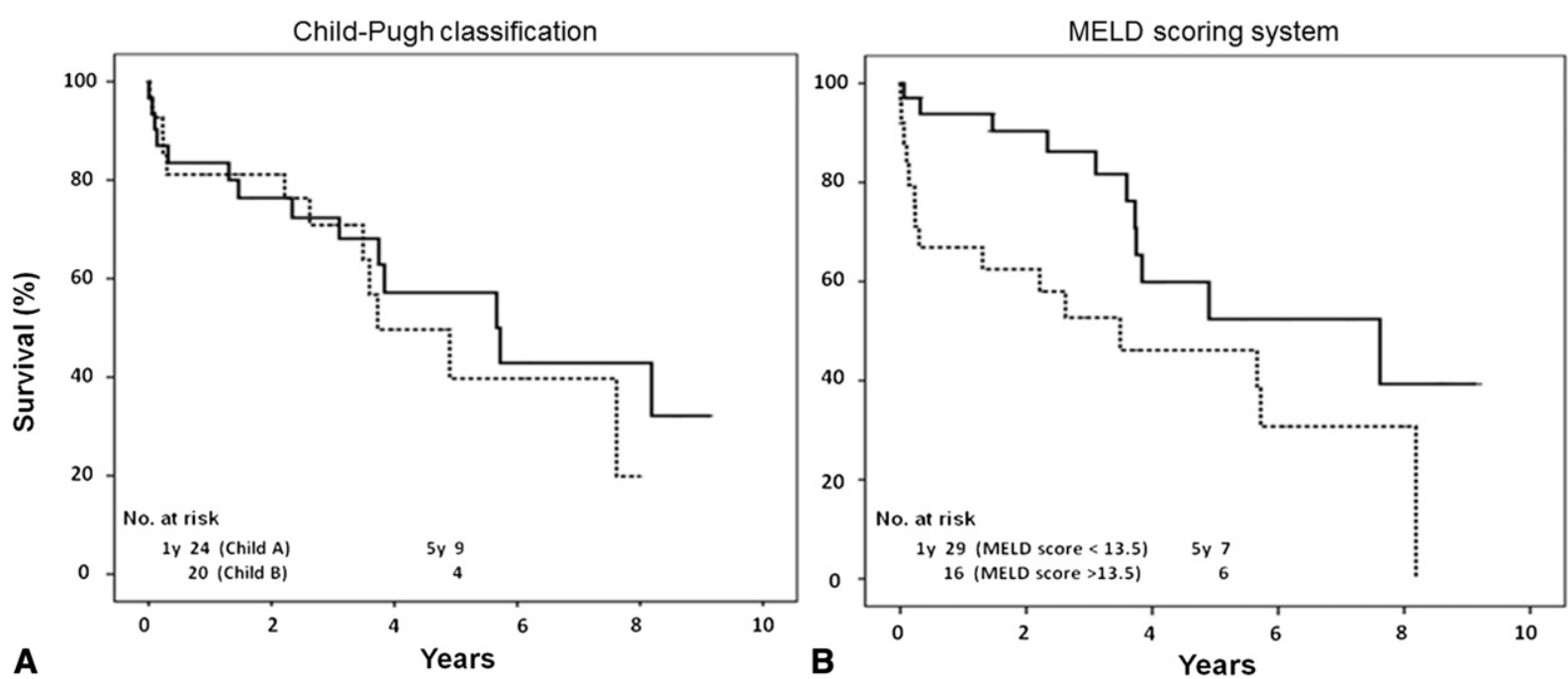

FIGURE 1. A, Kaplan-Meier survival curves. Child-Pugh class A group versus Child-Pugh class B group (solid line, Child-Pugh class A; dashed line, Child-Pugh class B; log-rank, $P=.60)$. B, Kaplan-Meier survival curves for Model for End-Stage Liver Disease $(M E L D)$ score $<13.5$ group versus MELD score $>13.5$ group (solid line, MELD score $<13.5$; dashed line, MELD score $>13.5$; $\log$-rank, $P=.027$ ).

at 5 years. $^{8}$ Recent immunosuppressive regimens have included a combination of a calcineurin inhibitor and corticosteroids/mycophenolate mofetil/sirolimus. Although the current immunosuppressive regimens have played an important role in improving the outcomes of transplantation, these medications are associated with exacerbation of systemic and metabolic disorders, including, but not limited to, hypertension, diabetes mellitus, hyperlipidemia, and obesity. ${ }^{9}$ Johnston and colleagues ${ }^{10}$ reported that liver allograft recipients have a greater risk of cardiovascular death and ischemic events than an age- and gender-matched population. With the increased life expectancy and increased risks of cardiovascular diseases for liver allograft recipients, it would be reasonable to assume that the number of liver recipients in need of cardiac surgery could be increasing.

Cardiac surgery for patients with liver dysfunction is still challenging. It has generally been accepted that patients in Child-Pugh class $\mathrm{C}$ are contraindicated for cardiac surgery. ${ }^{11}$ Our institution has been following that surgical indication; subsequently, only patients with Child-Pugh class A or B were involved in the present study. Cardiac surgery for patients with cirrhosis has been well documented. Reportedly, the operative mortality of patients with Child-Pugh class $\mathrm{A}, \mathrm{B}$, and $\mathrm{C}$ has ranged from $3 \%$ to $5 \%, 35 \%$ to $50 \%$, and $70 \%$ to $100 \%$, respectively. ${ }^{12-14}$ In the present study, the overall survival rate was $82.4 \% \pm 5.1 \%$ at 1 year and $50.2 \% \pm 8.2 \%$ at 5 years, comparable to that of other investigator. ${ }^{15}$ During the follow-up period, only 1 patient $(1.6 \%)$ lost the liver allograft and required subsequent liver repeat transplantation. We believe that the early and late outcomes in the present study were acceptable.

Realizing that patients with cirrhosis and those with a functioning liver allograft represent different populations, the present study has showed that the overall in-hospital mortality was $6.6 \%$ and for Child-Pugh class A and B was $6.1 \%$ and $7.1 \%$, respectively, with no statistically significant difference. In addition, the Kaplan-Meier survival analysis showed no significant difference in the survival rates between the Child-Pugh class $\mathrm{A}$ and $\mathrm{B}$ groups (Child-Pugh class A, $83.5 \% \pm 6.8 \%$ at 1 year and $57.2 \% \pm 10.3 \%$ at 5 years vs Child-Pugh class B, $81.1 \% \pm 7.6 \%$ at 1 year and $39.7 \% \pm 13.2 \%$ at 5 years; log-rank, $P=.603$ ). This discrepancy between patients with cirrhosis and patients with a functioning liver allograft might be a limitation of the Child-Pugh classification system, which uses arbitrary cutoff values.

However, the MELD score system makes an incremental contribution to the score for each 1-point increase in the factors. Therefore, it can be expected that the MELD score will increase the precision in predicting postoperative mortality. The MELD score (range, 6 to 40), which is a linear regression model calculated from the serum bilirubin, international normalized ratio of prothrombin time, and serum creatinine, was adopted in liver transplantation for allocation purposes. ${ }^{16}$ The MELD score is a well-known good predictor of waiting list mortality and post-liver transplant mortality and has recently been used to predict perioperative mortality in the different categories of surgery. It has been reported that the MELD score is the most precise single predictor of perioperative mortality. ${ }^{11}$ However, it has not been well documented in the field of cardiac surgery. In the present study, we sought to establish a cutoff value for the MELD score to predict the mortality of cardiac surgery for patients with a functioning liver allograft. The present study showed that the mean of the preoperative MELD score for the patients who died postoperatively was 
significantly greater than that of the other patients. From the statistical analysis, the optimal cutoff value was identified as 13.5. This cutoff value is similar to those previously reported to predict the surgical mortality of patients with cirrhosis (eg, MELD score $>13$ ). ${ }^{14}$ To reconfirm the adequacy of the cutoff value (ie, 13.5), an additional Kaplan-Meier analysis was performed by regrouping the cohort into 2 groups (ie, patients with a MELD score of $>13.5$ or $<13.5$ ). This analysis successfully showed that the survival rate of the group with a MELD score less than 13.5 was significantly greater than that of those with a MELD score greater than 13.5 (log-rank, $P=.027$ ).

The study limitations included the retrospective nature and analysis and the relatively small number of patients. However, the present study is 1 of the largest series of cardiac surgery in liver transplant recipients. In addition, we acknowledge that the sensitivity and specificity of the cutoff value of 13.5 of the MELD score was only $56 \%$ and $68 \%$, respectively. The AUC was 0.633 , considered of "low accuracy." It is generally accepted that an AUC of 0.9 to 1.0 represents high accuracy, an AUC of 0.7 to 0.9 as moderate accuracy, and AUC of 0.5 to 0.7 as low accuracy. ${ }^{17}$ However, the cutoff value of 13.5 was shown to predict late mortality in the present study with statistical significance. Therefore, we believe that the preoperative MELD score should be 1 of the important parameters to evaluate when assessing patients with liver issues for surgery. Regarding the postoperative liver complications, although the new onset of ascites and encephalopathy during the postoperative in-hospital period was likely related to the cardiac surgery, it is unclear whether the other liver complications that occurred in the chronic phase resulted from cardiac surgery or simply reflected the natural history of the liver transplant recipients. Also, although postoperative encephalopathy was diagnosed by the liver transplantation team, we acknowledge that it is sometimes difficult and confusing to differentiate hepatic encephalopathy from common postoperative delirium.

\section{CONCLUSIONS}

We reviewed our experience with the surgical treatment and outcomes of cardiac surgery for patients with a functioning liver allograft. The surgical outcomes in the present study were acceptable in terms of both the in-hospital and the late outcomes. Preoperative encephalopathy and pulmonary hypertension were independent predictors of late mortality. The optimal cutoff value of the preoperative MELD score was identified as 13.5 for predicting surgical mortality. The preoperative MELD score could possibly be useful to predict surgical mortality for patients with liver dysfunction who are undergoing cardiac surgery.

\section{References}

1. Merion RM. Current status and future of liver transplantation. Semin Liver Dis. 2010;30:411-21.

2. Dec GW, Kondo N, Farrell ML, Dienstag J, Cosimi AB, Semigran MJ. Cardiovascular complications following liver transplantation. Clin Transplant. 1995;9: 463-71.

3. Fouad TR, Abdel-Razek WM, Burak KW, Bain VG, Lee SS. Prediction of cardiac complications after liver transplantation. Transplantation. 2009;87:763-70.

4. Gangappa S, Kokko KE, Carlson LM, Gourley T, Newell KA, Pearson TC, et al. Immune responsiveness and protective immunity after transplantation. Transpl Int. 2008;21:293-303.

5. Schneider PD. Preoperative assessment of liver function. Surg Clin North Am. 2004;84:355-73.

6. Riordan SM, Williams R. Treatment of hepatic encephalopathy. N Engl J Med. 1997;337:473-9.

7. Durack DT, Lukes AS, Bright DK. New criteria for diagnosis of infective endocarditis: utilization of specific echocardiographic findings. Duke Endocarditis Service. Am J Med. 1994;96:200-9.

8. Futagawa Y, Terasaki PI, Waki K, Cai J, Gjertson DW. No improvement in longterm liver transplant graft survival in the last decade: an analysis of the UNOS data. Am J Transplant. 2006;6:1398-406.

9. Bianchi G, Marchesini G, Marzocchi R, Pinna AD, Zoli M. Metabolic syndrome in liver transplantation: relation to etiology and immunosuppression. Liver Transpl. 2008; 14:1648-54.

10. Johnston SD, Morris JK, Cramb R, Gunson BK, Neuberger J. Cardiovascular morbidity and mortality after orthotopic liver transplantation. Transplantation. 2002;73:901-6.

11. Friedman LS. Surgery in the patient with liver disease. Trans Am Clin Climatol Assoc. 2010;121:192-204.

12. Hayashida N, Shoujima T, Teshima H, Yokokura Y, Takagi K, Tomoeda H, et al. Clinical outcome after cardiac operations in patients with cirrhosis. Ann Thorac Surg. 2004;77:500-5.

13. Suman A, Barnes DS, Zein NN, Levinthal GN, Connor JT, Carey WD. Predicting outcome after cardiac surgery in patients with cirrhosis: a comparison of ChildPugh and MELD scores. Clin Gastroenterol Hepatol. 2004;2:719-23.

14. Modi A, Vohra HA, Barlow CW. Do patients with liver cirrhosis undergoing cardiac surgery have acceptable outcomes? Interact Cardiovasc Thorac Surg. 2010; 11:630-4.

15. Deb SJ, Mullany CJ, Kamath PS, Dearani JA, Daly RC, Orszulak TA, et al. Cardiac surgery in kidney and liver transplant recipients. Mayo Clin Proc. 2006;81:917-22.

16. Wiesner R, Edwards E, Freeman R, Harper A, Kim R, Kamath P, et al. Model for End-stage Liver Disease (MELD) and allocation of donor livers. Gastroenterology. 2003; 124:91-6.

17. Akobeng AK. Understanding diagnostic tests 3: receiver operating characteristic curves. Acta Paediatr. 2007;96:644-7. 